\title{
EVALUATION OF THE RESULTS OF CLOSED INTERLOCKING INTRAMEDULLARY NAILING IN FRACTURE SHAFT OF HUMERUS
}

\author{
Zeeshan Muzahid ${ }^{1}$,Kammela Hareesh ${ }^{2}$, M. Srikant ${ }^{3}$ \\ 1 Postgraduate, Department of Orthopaedics, Konaseema Institute of Medical Sciences, Amalapuram. \\ ${ }^{2}$ Postgraduate, Department of Orthopaedics, Konaseema Institute of Medical Sciences, Amalapuram. \\ ${ }^{3}$ Resident, Department of Orthopaedics, Konaseema Institute of Medical Sciences, Amalapuram.
}

ABSTRACT
BACKGROUND
Intramedullary interlocking nailing has become the standard method of management for closed diaphysis fracture of long
bones. Being close to the weight bearing axis, an intramedullary nail is at a mechanical advantage over other fracture stabilisation
devices such as plates, external fixator and casts. The present clinical study is an evaluation of results in the internal fixation of
fractures shaft of humerus by intramedullary interlocking nailing.

\section{MATERIAL AND METHOD}

The present study will be conducted in the Department of Orthopaedics, KIMS Hospital, Amalapuram, during the period June 2012 to June 2014. In this study cases of fracture shaft of humerus belonging to various age groups will be selected and treated by closed interlocking nail. In all the cases thorough history will be taken to assess the mechanism and nature of injury and quality radiographs were taken to know the geometry of fracture.

\section{RESULTS AND CONCLUSION}

This is a clinical study done between June 2012 to June 2014 involving 13 antegrade reamed closed humeral shaft fractures in the Department of Orthopaedics, Konaseema Institute of Medical Sciences, Amalapuram. In this series, Road Traffic Accidents accounted for 11 cases and 2 cases in accidental fall category. There is a male preponderance of the fracture representing 10 patients and 3 female cases, which works out to a ratio of 3.5:1. Of the 92 patients with fracture shaft of humerus 13 were treated with interlocking nail in Orthopaedics Department are discussed in this study their case reports. Followup was possible for the above all 13 cases, 11 patients showed good clinical and radiological fracture union. Post-operatively two cases have shown delayed union.

\section{KEYWORDS}

Closed Interlocking, Intramedullary Nailing, Fracture Shaft of Humerus.

HOW TO CITE THIS ARTICLE: Muzahid Z, Hareesh K, Srikant M. Evaluation of the results of closed interlocking intramedullary nailing in fracture shaft of humerus. J. Evolution Med. Dent. Sci. 2016;5(80):5950-5955, DOI: 10.14260/jemds/2016/1344

\section{INTRODUCTION}

The surgical specialty of orthopaedics has come a long way since the day of Nicolas Andry (1741). The discovery of X-rays by Roentgen and the introduction of the usage of Plaster of Paris by Antonius Mathijsen in 1852 revolutionised the diagnosis and management of orthopaedic conditions. There is sudden surge of orthopaedic cases firstly by the two World wars, and of late by the road traffic accidents which is on the rise both in the developed and the developing countries.

Polytrauma, multiple fractures and high velocity injuries severely exposed the limitation of the conventional treatment in orthopaedics, as the fracture patterns are bizarre and complicated necessitating early surgical intervention.

Road traffic accidents ranks first among all fatal accidents followed by industrial accidents, falls, railway accidents and assaults leading to high incidence of morbidity and mortality.

Financial or Other, Competing Interest: None.

Submission 02-05-2016, Peer Review 28-05-2016,

Acceptance 03-06-2016, Published 05-10-2016.

Corresponding Author:

Dr. Zeeshan Muzahid

Postgraduate,

Department of Orthopaedics,

Konaseema Institute of Medical Sciences,

Amalapuram.

E-mail: anand_kims@yahoo.co.in

DOI: $10.14260 /$ jemds $/ 2016 / 1344$
These road traffic accidents and the injuries caused by them are of great importance not only to the many thousands who suffer from them each year but also to the medical, social services and occupational services because of the enormous morbidity and demands made on them by those injured. So now the emphasis is on to restore and recuperate those injured as early as possible, so that they can regain the normal or near normal anatomical function and attend to their activities of daily life and occupation by means of early internal fixation of fractures and proper rehabilitation.

The vascular supply of the bone is the basis of all fracture healing. The ideal goal of fracture treatment is to obtain solid union of the fracture in the most acceptable anatomical position compatible with maximal functional return to the extremity.

In the words of Sir John Charnely fracture shaft of humerus is perhaps the easiest of the major long bones to treat by conservative methods. Most of the fractures of the shaft of the humerus treated conservatively are clinically united by about six weeks, which was known as far back as the time of Hippocrates. Unfortunately, not all the fractures of shaft of humerus behave in this way. Union may not be clinically firm at six weeks and if not given appropriate treatment the fracture can go into a state of established nonunion. The need for other methods of fracture treatment may also arise when there are compound injuries and the use of external fixator may be valuable if the soft tissue damage is 
extensive. Internal fixation may be required for patients with more than one fracture in the same limb for double fractures or where there are multiple fractures of humerus or multiple fractures elsewhere in the body and associated neurovascular injuries.

Internal fixation of fracture shaft of humerus allows ready access for nursing care in patients with multiple injuries and associated comorbid conditions. And also restrictive bandages and splints are avoided, early mobilisation of the patients and his limbs helps to prevent fracture disease.

Intramedullary interlocking nailing has become the standard method of management for closed diaphyseal fracture of long bones. Being close to the weight bearing axis, an intramedullary nail is at a mechanical advantage over other fracture stabilisation devices such as plates, external fixator and casts. The intramedullary nailing can be inserted in reamed or unreamed fashion with its own merits and demerits. The initial unlocked design was modified to allow proximal and distal interlocking. This further improved stability and expanded the indication for the nailing to include comminuted fractures and more proximal and distal fractures. The technique is now well established in the management of fractures of long bones of the lower limb. There are many previous studies done by McCormack Cox MA,(1) Crates J,(2) M Ajmal et al,(3) George Petsatodes et al,(4) Muralidhar BM et al(5) and M.J.G. Blyth(6) et al.

The present clinical study is an evaluation of results in the internal fixation of fractures shaft of humerus by intramedullary interlocking nailing done in the Department of Orthopaedics, Konaseema Institute of Medical Sciences and Research Foundation, Amalapuram.

\section{MATERIALS AND METHODS}

The present study will be conducted in the Department of Orthopaedics, KIMS Hospital, Amalapuram during the period June 2012 to June 2014. In this study, cases of fracture shaft of humerus belonging to various age groups will be selected and treated by closed interlocking nail. In all the cases thorough history will be taken to assess the mechanism and nature of injury and quality radiographs were taken to know the geometry of fracture. After the thorough preoperative assessment and necessary consent, the cases will be taken up for surgery.

\section{MATERIALS}

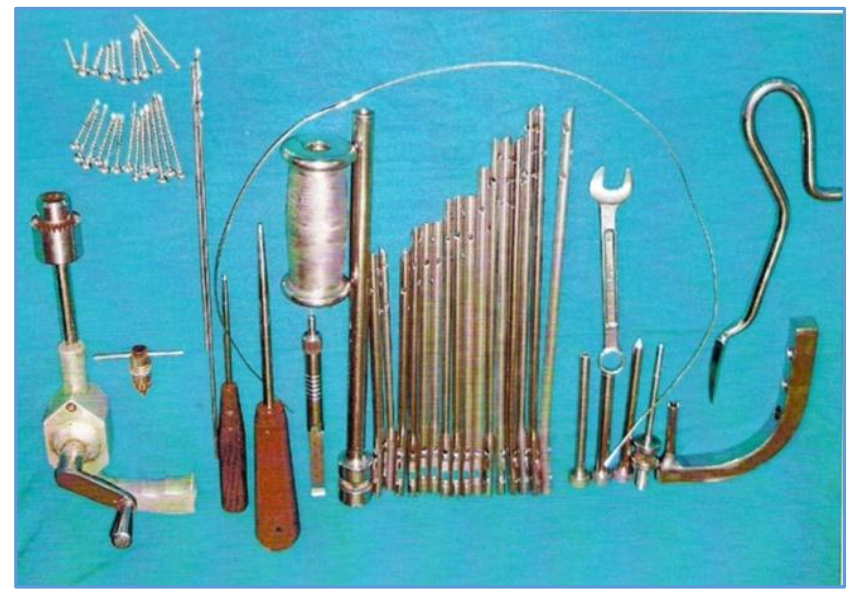

Instruments for Interlocking Humerus
1. General instruments for orthopaedic surgery (Like surgical blades with handles, haemostatic forceps, dissection forceps, retractors, scissors, etc.)

2. Special instruments like bone awl; jig with sleeves; guide wire; reamers 6, 7, 8 sizes; drill bit $4 \mathrm{~mm}$ size; mallet.

3. Interlocking nails $6,7,8$ sizes with locking screws.

4. Depth gauge.

5. Power drill with power source.

6. Screw drivers.

7. Suture materials, etc.

\section{Method of Closed Intramedullary Interlocking Nail Pre-Operative Plan}

1. General condition of the patient is stabilised.

2. Preoperative investigations like $\mathrm{Hb}, \mathrm{BT}, \mathrm{CT}, \mathrm{TC}, \mathrm{DC}, \mathrm{ESR}$ blood grouping and typing, blood sugar, blood urea, serum creatinine, ECG were done.

3. X-rays were carefully examined for fracture pattern, degree of comminution and approximate estimation of diameter and length of nail.

4. Fractures classified as per A.O. classification.

\section{Anaesthesia}

1. General anaesthesia, or

2. Interscalene block supplemented with short general anaesthesia.

\section{Position of the Patient}

The patient was positioned supine with pillow below the shoulder girdle with patient head turned to opposite side to maximise exposure of shoulder under image intensifier.

Reduction of fracture is obtained by closed manipulation of fracture under image intensifier and checked both in antero-posterior and lateral views.

\begin{abstract}
Approach
A longitudinal skin incision starting from the most lateral point of the acromion and extending distally, centered over the tip of the greater tuberosity. The incision should not extend more than $4-5 \mathrm{~cm}$ in deltoid muscle in order to avoid damage to axillary nerve.
\end{abstract}

\section{PROCEDURE}

In all our cases, procedure will be carried out by antegrade technique. Entry point is made with bone awl medial to the tip of greater tuberosity. The bone awl is advanced by rotatory movements until it is seated within the humeral head.

The position of bone awl is confirmed by image intensifier by taking anteroposterior and lateral views. The position of bone awl is adjusted under image control, so that entry portal was centered both in anteroposterior and lateral views to ensure that nail will be in the mid plane of humerus.

Then $2.4 \mathrm{~mm}$ guide wire is advanced through the entry point into the medullary cavity. Fracture site is reduced and guide wire is advanced from proximal to distal fragment and confirmed by image intensifier by anteroposterior and lateral views. We advance the guide wire into distal fragment until tip is 1 to $2 \mathrm{~cm}$ proximal to the olecranon fossa. 


\section{Measuring the Required Nail Length}

For measuring required nail length, we use guide wire method. In this method with distal end of guide wire 1 to 2 $\mathrm{cm}$ proximal to olecranon fossa. A second guide wire of same length as that of intramedullary guide wire is kept at $1 \mathrm{~cm}$ below the entry point.

Now the length of the second wire, which is overlapping the first guide wire is calculated.

\section{Reaming Technique}

The entire humerus is reamed over the $2.4 \mathrm{~mm}$ guide wire in $0.5 \mathrm{~mm}$ increments until the desired diameter is achieved.

\section{Nail Insertion}

The selected nail is attached to the proximal jig with conical bolt with handle pointing away from the patient and proximal curvature of nail pointed laterally.

Nail is driven over the guide wire by gentle movements of the jig up to fracture site, fracture reduction was confirmed in both antero-posterior and lateral views by image intensifier and advanced gently over the fracture site to the distal fragment to avoid comminution at fracture site.

Guide wire was removed and humeral driver was attached to the jig and nail was advanced by gentle tapping until it is 1 to $2 \mathrm{~cm}$ proximal to olecranon fossa.

\section{Proximal Locking}

Proximal locking is done with the help of jig; $8 \mathrm{~mm}$ drill sleeve was passed through proximal drill guide and pushed to the bone by small skin incision; $2.7 \mathrm{~mm}$ humeral drill sleeve is passed through $8 \mathrm{~mm}$ sleeve; $2.7 \mathrm{~mm}$ trocar is used to make dimple on outer cortex; $2.7 \mathrm{~mm}$ drill bit is used to drill from lateral to medial side. Drill is removed, size of screw is determined by humeral screw length gauge.

The selected $4 \mathrm{~mm}$ fully threaded screw is inserted.

\section{Distal Locking}

Distal locking is done by free hand technique. Anterior approach is used where small skin incision is given and biceps muscle is split by blunt dissection. Distal hole drilled anteroposterior direction with $2.7 \mathrm{~mm}$ drill bit under image guidance. Appropriate screw length was measured by humeral screw length gauge and desired $4 \mathrm{~mm}$ screw was inserted. Both incisions are thoroughly irrigated with saline, hydrogen peroxide and closed in layers.

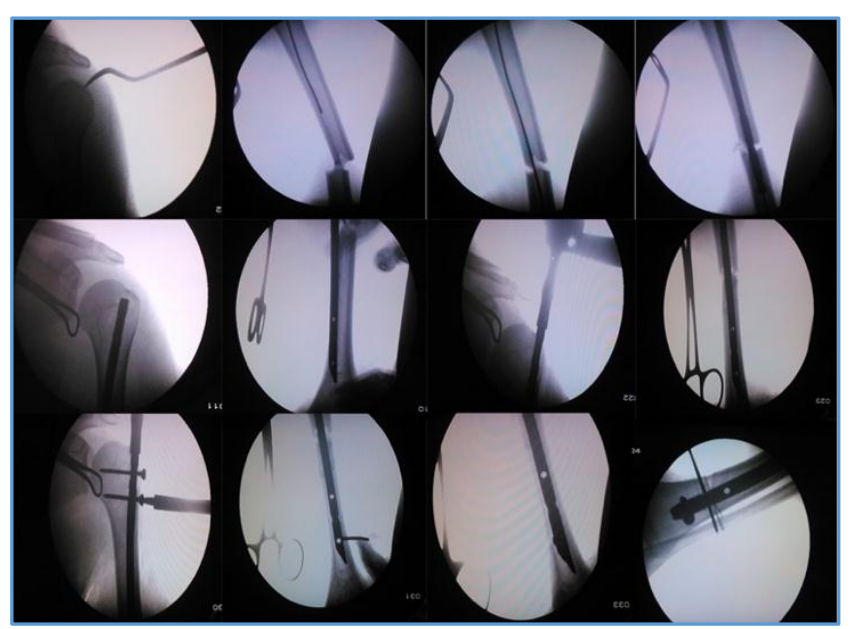

C-ARM Images of Humerus Nailing

\section{Post-Operative Management}

Patients are given IV antibiotics for 3 days followed by oral antibiotics. Wound inspection is done on second postoperative day.

Patients are encouraged to do active movements of ipsilateral shoulder, elbow and hand from $2^{\text {nd }}$ post-operative day. Arm sling pouch is given to support the limb. Suture removal is done on $10^{\text {th }}$ post-operative day. Patients are thoroughly educated about role of physiotherapy in postoperative period.

\section{Follow-Up}

The cases were followed at regular intervals of 8 weeks, 12 weeks and 16 weeks and were assessed according to DASH (Disability of Arm Shoulder and Hand) criteria.

\section{Grading Excellent}

1. Clinically sound union and radiologically good union.

2. Full range or near normal active range of motion at shoulder and elbow.

3. No deformity and disability.

4. No post-operative complication (Radial nerve injury, infection, etc.).

5. DASH score 0-25.

\section{Good}

1. Clinically and Radiologically sound union.

2. $70 \%-90 \%$ of active range of motion at shoulder and elbow, no shoulder, elbow stiffness.

3. No deformity and disability.

4. No post-operative complication.

5. DASH score 26-50.

\section{Fair}

1. Clinically and Radiologically delayed union.

2. $50 \%$ to $70 \%$ of active range of motion.

3. Mild degrees of post-operative complications like superficial infection.

4. No deformity and disability.

5. DASH score 51-75.

\section{Poor}

1. Clinically and radiologically nonunion.

2. Less than $50 \%$ of active range of motion at shoulder and elbow.

3. Post-operative complications.

4. Deformity and disability.

5. DASH score 76-100.

\section{ANALYSIS OF RESULTS}

The analysis of results of closed reduction and internal fixation of the fractures of shaft of humerus by intramedullary interlocking nail was carried out in the Department of Orthopaedics, Konaseema Institute of Medical Sciences, Amalapuram.

The total number of trauma cases admitted in the Department of Orthopaedics during the period from Jun 2012 to Jun 2014 was 1215 , out of these cases 556 were lower limb and 320 upper limb fractures of which 92 were humerus fractures. Incidence of fracture shaft of the humerus during this period was $2.8 \%$. 
Of the 92 cases of humerus fractures which were admitted in the hospital 22 cases were supracondylar fractures in children requiring surgery, 21 cases were around the proximal humerus involving the surgical neck, 16 cases were around distal end of humerus in adults at or below the level of olecranon fossa, 9 of them with intra-articular extension.

Of all humerus fractures 33 cases had fracture shaft of humerus, out of which 7 cases were having fracture either very near to the surgical neck of humerus or were less than three centimetres from olecranon fossa into the shaft; 9 cases were treated with open reduction and internal fixation with DCP plate either because of radial nerve palsy pre-operatively or due to narrow marrow cavity; 1 case was treated with bridge plating due to comminution, 3 cases were treated with external fixator due to open contaminated injuries. During these two years period, 13 cases of fracture shaft of humerus were treated with intramedullary interlocking nailing technique.

In this series, there were 10 male and 3 female patients. Age incidence varied from 18 years to 60 years and 4 cases were in the age group of 18-30 years and 6 cases were in the age group of 30 to 50 years; 3 cases were in age group 50-60 years. The left humerus was involved in 5 cases and remaining 8 cases were on the right side.

All 13 cases are closed fractures. Type A3 fractures were common representing 6 cases, Type A2 were 2 cases, Type B3 were 2 cases, Type A1 were 2 cases, Type B2 was 1 case.

In this series all fractures are around middle $1 / 3^{\mathrm{rd}}$ junction of shaft of humerus, except one which was in upper $1 / 3^{\text {rd }}$ junction. Fractures in lower $1 / 3^{\text {rd }}$ and those having preoperative radial nerve palsy were preferably treated with open reduction and internal fixation with DCP plating.

The cause of fracture was RTA in 11 cases and fall from tree in 1 case. Fall at home in one patient.

\section{ANALYSIS OF 13 CASES}

\begin{tabular}{|c|c|c|}
\hline Age in Years & No. of Cases & Percentage \\
\hline $20-30$ & 4 & 31 \\
\hline $30-50$ & 6 & 46 \\
\hline $50-65$ & 3 & 23 \\
\hline \multicolumn{3}{|c|}{ Table 1: Age Incidence of Fracture Shaft Humerus } \\
Treated by Intramedullary Interlocking Nail \\
\hline
\end{tabular}

\begin{tabular}{|c|c|c|}
\hline Sex & No. of Cases & Percentage \\
\hline Male & 10 & 77 \\
\hline Female & 3 & 23 \\
\hline \multicolumn{2}{|c|}{ Table 2: Sex Incidence } \\
\hline
\end{tabular}

\begin{tabular}{|c|c|c|}
\hline Nature & No. of Cases & Percentage \\
\hline Simple & 13 & 100 \\
\hline \multicolumn{3}{|c|}{ Table 3: Nature of Fracture } \\
\hline
\end{tabular}

\begin{tabular}{|c|c|c|}
\hline Side & No. of Cases & Percentage \\
\hline Left & 5 & 38 \\
\hline Right & 8 & 62 \\
\hline \multicolumn{3}{|c|}{ Table 4 } \\
\hline
\end{tabular}

\begin{tabular}{|c|c|c|}
\hline Side & No. of Fracture & Percentage \\
\hline Upper third & 1 & 8 \\
\hline Middle third & 12 & 92 \\
\hline Lower third & 0 & 0 \\
\hline \multicolumn{3}{|c|}{ Table 5 } \\
\hline
\end{tabular}

\begin{tabular}{|c|c|c|}
\hline A.0. Type & No. of Cases & Percentage \\
\hline A1 & 2 & 15 \\
\hline A2 & 2 & 15 \\
\hline A3 & 6 & 46 \\
\hline B1 & 1 & 8 \\
\hline B2 & 2 & 15 \\
\hline B3 & & \\
\hline C1 & & \\
\hline C2 & Table 6 \\
\hline C3 & \multicolumn{2}{|c|}{} \\
\hline \multicolumn{2}{|c|}{} \\
\hline
\end{tabular}

\begin{tabular}{|c|c|c|}
\hline Type of Complication & No. of Cases & Percentage \\
\hline $\begin{array}{c}\text { Restriction of ROM of } \\
\text { shoulder }\end{array}$ & 4 & 30 \\
\hline Infection & 0 & 0 \\
\hline Bursitis & 0 & 0 \\
\hline Subacromial impingement & 0 & 0 \\
\hline Delayed union & 2 & 16 \\
\hline Non-union & 0 & 0 \\
\hline \multicolumn{2}{|c|}{ Table 7: Post-Operative Complications } \\
\hline
\end{tabular}

\begin{tabular}{|c|c|}
\hline Total No. of Cases & $\mathbf{1 3}$ \\
\hline Cases follow-up for 24 weeks & 8 \\
\hline Follow-up for 16 weeks & 5 \\
\hline Deaths & Nil \\
\hline Table 8: Evaluation of Cases Treated by Interlocking Nail \\
\hline
\end{tabular}

\begin{tabular}{|c|c|c|}
\hline Results & No. of Cases & Percentage \\
\hline Excellent & 8 & 61 \\
\hline Good & 5 & 38 \\
\hline Poor & 0 & 0 \\
\hline \multicolumn{3}{|c|}{ Table 9 } \\
\hline
\end{tabular}
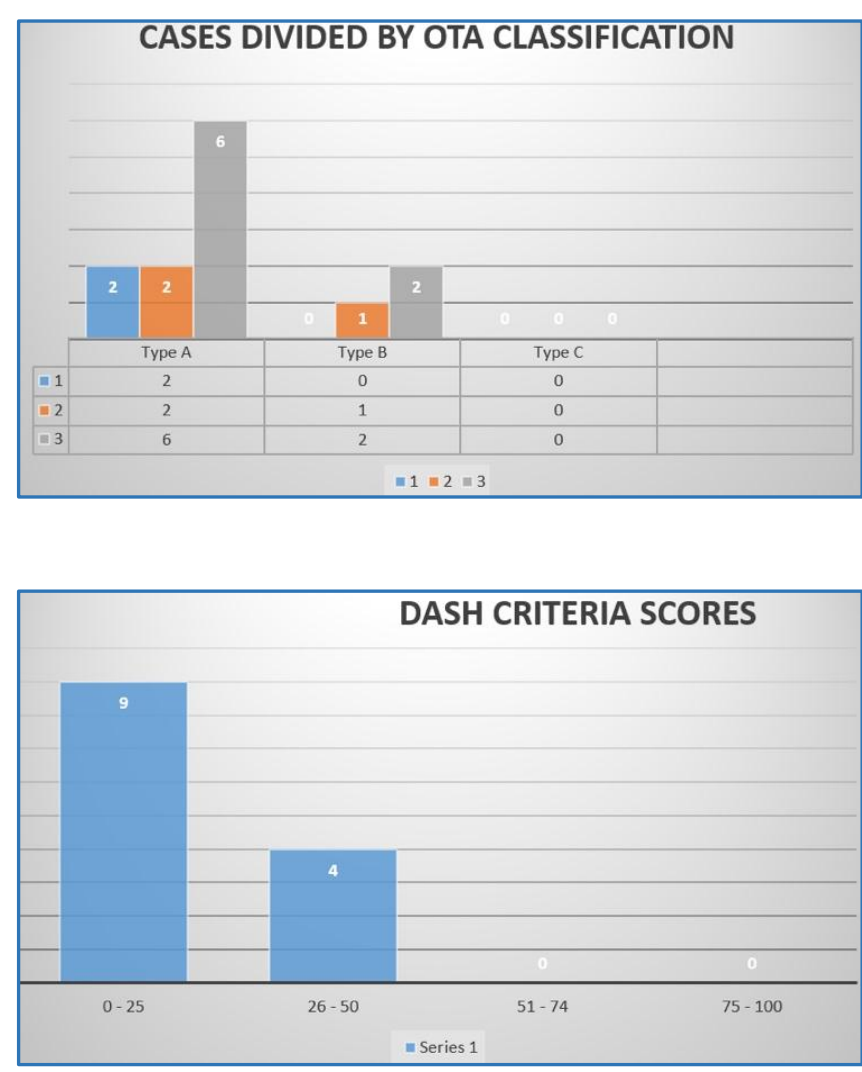


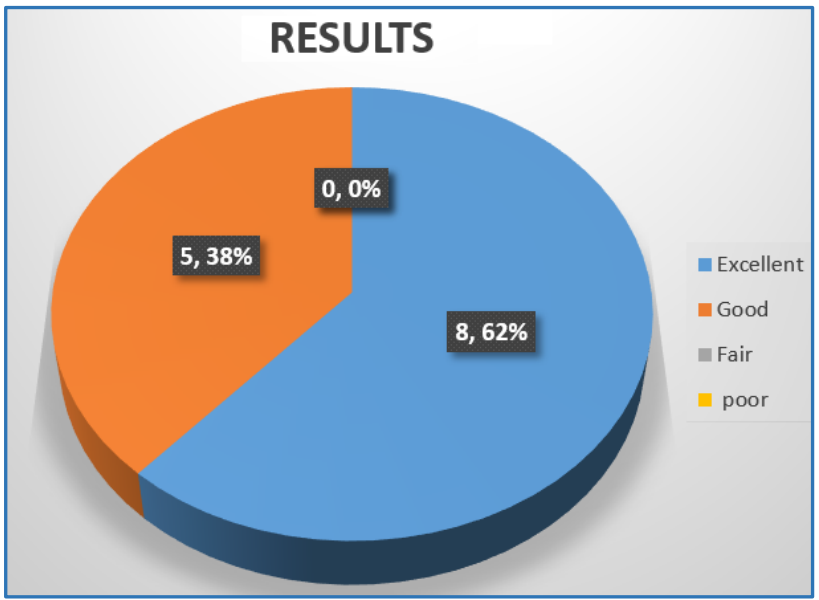

\section{DISCUSSION}

Fractures of the shaft of humerus account for $2.8 \%$ of all fractures treated. Historically humeral shaft fractures have been classified based on fracture location, fracture pattern, associated soft tissue injuries and quality of bone. In this study, AO classification has been taken to classify the fractures.

Many authors have documented the general good outcome that occurs after compression plate fixation, which is still considered the gold standard for operative treatment of acute humeral shaft fractures. Though plate fixation has given high rates of union, it involves extensive soft tissue stripping, potential injury to radial nerve and poor fixation in osteoporotic bone. Later flexible nails of many varieties were used. The advantages of intramedullary interlocking nailing are minimal surgical exposure, better biomechanical fixation, minimal disturbances of soft tissues and early mobilisation of neighbouring joints.

The technique of interlocking nailing represents the newer approach of the treatment of humeral fractures. Interlocking nailing also avoids complications like lack of rotational control, migration of nail and requirement of supplementary bracing. The Seidel nail was the first nail to be tested clinically.

This is a clinical study done between June 2012 to June 2014 involving 13 antegrade reamed closed humeral shaft fractures in the Department of Orthopaedics, Konaseema Institute of Medical Sciences, Amalapuram.

In this series, Road Traffic Accidents accounted for 11 cases and 2 cases in accidental fall category. There is a male preponderance of the fracture representing 10 patients and 3 female cases which works out to a ratio of 3.5:1.

The occurrence of the fracture in productive and active age group is very high representing 10 cases who are in age group of 20 to 50 years. All these cases had road traffic accidents indicating high velocity injuries as a pre-requisite for shaft of humerus fractures. In the present study, low velocity injuries had caused fractures of humeral shaft only in old age who had osteoporosis.

Most of the fractures occurred on the right side which account for 8 cases representing $62 \%$ and the left side, 5 cases representing 38\%. Majority of the fractures occurring in the middle third shaft of humerus were fixed by IMIL nail. These require stable fixation and proper reduction for early fracture union and to avoid damage to the radial nerve, which may occur during manipulation and casing in conservative methods. The average period between fracture and surgical fixation was 4.4 days in the present study, most of this delay was attributable to comorbid conditions of the patient and holidays. All the fractures were easily reducible even after this delay with implication of proper traction and manipulation techniques intra-operatively.

All the nails were inserted through antegrade technique with entry point just medial to greater tuberosity. All these fractures were fixed using a humeral nail of sizes $6,7,8 \mathrm{~mm}$ diameter and of appropriate length calculated intraoperatively.

In 7 patients the operated limb was immobilised for two days and active-assisted shoulder mobilisation was started from $3^{\text {rd }}$ postoperative day. Immediate mobilisation was started in 3 patients. In 3 patients mobilisation of the shoulder was delayed due to associated injuries of both bones fracture in one patient and tibial fractures in two patients. The duration of post-operative prophylactic intravenous antibiotics was 3 days. None of our patients had any infections. Average hospitalisation of each patient in this study is 11 days. Out of 13 patients 8 patients were followed up for a minimum of 6 months postoperatively and maximum up to 2 years, 3 cases were followed up for 4 months and two cases were followed for 12 weeks. During post-operative period patients were encouraged to do physiotherapy, Calcium and Vitamin D3 were administered in old age persons.

\section{Radiological Union}

Radiological union was defined as the presence of bridging callus of the cortices in two orthogonal views. Delayed union was defined as failure of fracture union to occur by 4 months. Nonunion was defined as failure of fracture union by nine months.

Out of 13 cases, 11 cases showed normal union within 3 to 4 months from the day of surgery. Two cases showed signs of delayed union even after 4 months. One case had associated fracture of both bones forearm. Both the patients had satisfactory intra-operative reduction and fixation. One of the cases was intervened with infusion of PRP (Platelet Rich Plasma) into the fracture site, which later showed normal fracture union. The other delayed union case has refused for further intervention and is being followed up.

Two cases had splintering of at the fracture site during nail insertion into distal fragment resulting in formation of small butterfly fragments. One case showed sound union in due date, while the other case had delayed union. Nail was short in few cases, i.e. its tip did not reach the $2 \mathrm{~cm}$ mark above olecranon, but even then they showed sound radiological union. Three cases had associated fractures, one had delayed union who even had both bones forearm fracture of the same limb, other two cases having tibia fractures had normal union. Overall union rate $84.6 \%$ and delayed union rate is $15.3 \%$.

This result is comparable to the union rate achieved by McCormack (89.48\%), Cox MA(1) $(87.9 \%)$, Crates J(2) $(94.5 \%)$, M Ajmal et al(3) (81\%), George Petsatodes et al(4) $(84.6 \%)$ and Muralidhar BM et al(5) (90.6\%).

The rate of delayed union in George Petsatodes et al study was $7.7 \%$ and in Muralidhar BM et al study was $9.37 \%$. Nonunion was reported in the studies of M Ajmal et al(3) (19\%), Cox MA (12.1\%). 


\section{Functional Outcome}

Almost all the previous studies showed concerns in antegrade intramedullary interlocking nailing of humeral shaft fractures regarding shoulder stiffness.

Previous reports have implicated injury to the rotator cuff, disruption of the articular cartilage or adhesive capsulitis as a result of antegrade entry. In the antegrade approach, it is essential to ensure that both the nail and proximal locking screws are deep to the cortex to reduce impingement. In the present study 4 out of 13 cases had shoulder stiffness, accounting for nearly $30 \%$. All the patients were above 50 years with comorbid conditions and lack of proper physiotherapy.

M J G Blyth ${ }^{6}$ et al reported radial nerve palsy in 5.8\% cases of antegrade nailing. Bell and Vander Griend reported $2.9 \%$ radial nerve palsy following plating of humeral shaft fractures. None of the cases in this present study had post-op radial nerve palsy.

All of the 13 cases had good-to-excellent functional rating. None of the patients underwent implant removal in our followup.

So our study shows that early fixation of fracture by closed reduction and internal fixation with intramedullary interlocking nail had resulted in early fracture union and early return to the functional and occupational activities. The study also coincides with results of George Petsatodes et al,(4) Muralidhar BM et al,(5) M Ajmal et al(3) 2001 and Bell MJ(7) study 2000 that interlocking nail is beneficial to the patients with fracture shaft of humerus, as it results in early return to functionability with near normal union.

\section{SUMMARY}

Of the 92 patients with fracture shaft of humerus, 13 were treated with interlocking nail in Orthopaedics Department, are discussed in this study their case reports.

Followup was possible for the above all 13 cases, 11 patients showed good clinical and radiological fracture union. Post-operatively, two cases have shown delayed union.

This study proves beyond doubt that closed reduction and internal fixation of fracture shaft of humerus by interlocking nail has distinct advantages of early perfect fracture stabilisation and early post-operative mobilisation of shoulder and elbow joints leading to early normal function of the upper limbs, provided the correct technique and principles of internal fixation are followed.

\section{CONCLUSION}

- Humeral interlocking nail is an effective means of fixation of acute humeral fractures. Intramedullary fixation is a simpler technique with minimal exposure and shorter operative time with less blood loss. The preservation of fracture haematoma, soft tissue and periosteum around the fracture that occurs with closed unreamed nailing has been proposed for high rates of union and good results with no risk of iatrogenic radial nerve palsy.

- Locked nailing is said to provide a rotationally stable fixation and avoid the tendency of various unlocked nails to back out.

- It is also of special value in open humeral fractures due to lower incidence of infections.

- Distraction at the fracture site should be avoided.

- Shoulder stiffness is a significant problem in antegrade nailing, which can be minimised if care is taken to prevent proximal protrusion of the nail, deep insertion of nail to prevent impingement, repair the rotator cuff properly and early institution of physiotherapy.

\section{REFERENCES}

1. Cox MA, Dolan M, Synnott K, et al. Closed interlocking nailing of humeral shaft fractures with the russell-taylor nail. Orthop Trauma 2000;14(5):349-53.

2. Crates J, Whittle PA. Antegrade interlocking nailing of acute humeral shaft fractures. Clin Orthop 1998;350: 40-50.

3. Ajmal M, O'Sullivan M, McCabe J, et al. Antegrade locked intramedullary nailing in humeral shaft fractures. Injury 2001;32(9):692-4.

4. Petsatodes G, Karataglis D, Papadopoulos P, et al. Antegrade interlocking nailing of humeral shaft fractures. J Orthop Sci 2004;9(3):247-52.

5. Muralidhar BM, Reddy MD. Surgical management of fracture shaft humerus with interlocking nail - a prospective study. Journal of Evolution of Medical and Dental Sciences 2013;2(41):7863-72.

6. Blyth MJ, Macleod CM, Asante DK, et al. Iatrogenic nerve injury with the russel-taylor humeral nail. Injury 2003;34(3):227-8.

7. Bell MJ, Beauchamp CG, Kellam JK, et al. The results of plating humeral shaft fractures in patients with multiple injuries. The Sunnybrook experience. J B J S Br 1985;67(2):293-6. 